Research

\title{
Glycemic penalty index for adequately assessing and comparing different blood glucose control algorithms
}

\author{
Tom Van Herpe ${ }^{1}$, Jos De Brabanter ${ }^{1,2}$, Martine Beullens ${ }^{3}$, Bart De Moor ${ }^{1}$ and Greet Van den \\ Berghe $^{4}$
}

\begin{abstract}
${ }^{1}$ Katholieke Universiteit Leuven, Department of Electrical Engineering (ESAT), Research Division SCD, Kasteelpark Arenberg 10, B-3001 Leuven (Heverlee), Belgium

${ }^{2}$ Hogeschool KaHo Sint-Lieven (Associatie K.U. Leuven), Dept. Industrieel Ingenieur, Gebroeders Desmetstraat 1, B-9000 Gent, Belgium ${ }^{3}$ Leuvens Universitair Dienstencentrum voor Informatica en Telematica (LUDIT), W. de Croylaan 52a, B-3001 Leuven (Heverlee), Belgium ${ }^{4}$ Katholieke Universiteit Leuven, Department of Intensive Care Medicine, University Hospital Gasthuisberg, Herestraat 49, B-3000 Leuven, Belgium

Corresponding author: Tom Van Herpe, tom.vanherpe@esat.kuleuven.be
\end{abstract}

Received: 8 Nov 2007 Revisions requested: 7 Dec 2007 Revisions received: 2 Jan 2008 Accepted: 26 Feb 2008 Published: 26 Feb 2008

Critical Care 2008, 12:R24 (doi:10.1186/cc6800)

This article is online at: http://ccforum.com/content/12/1/R24

(c) 2008 Van Herpe et al.; licensee BioMed Central Ltd.

This is an open access article distributed under the terms of the Creative Commons Attribution License (http://creativecommons.org/licenses/by/2.0), which permits unrestricted use, distribution, and reproduction in any medium, provided the original work is properly cited.

\begin{abstract}
Introduction Blood glucose (BG) control performed by intensive care unit (ICU) nurses is becoming standard practice for critically ill patients. New (semi-automated) 'BG control' algorithms (or 'insulin titration' algorithms) are under development, but these require stringent validation before they can replace the currently used algorithms. Existing methods for objectively comparing different insulin titration algorithms show weaknesses. In the current study, a new approach for appropriately assessing the adequacy of different algorithms is proposed.
\end{abstract}

Methods Two ICU patient populations (with different baseline characteristics) were studied, both treated with a similar 'nursedriven' insulin titration algorithm targeting BG levels of 80 to $110 \mathrm{mg} / \mathrm{dl}$. A new method for objectively evaluating BG deviations from normoglycemia was founded on a smooth penalty function. Next, the performance of this new evaluation tool was compared with the current standard assessment methods, on an individual as well as a population basis. Finally, the impact of four selected parameters (the average BG sampling frequency, the duration of algorithm application, the severity of disease, and the type of illness) on the performance of an insulin titration algorithm was determined by multiple regression analysis.

Results The glycemic penalty index (GPI) was proposed as a tool for assessing the overall glycemic control behavior in ICU patients. The GPI of a patient is the average of all penalties that are individually assigned to each measured BG value based on the optimized smooth penalty function. The computation of this index returns a number between 0 (no penalty) and 100 (the highest penalty). For some patients, the assessment of the BG control behavior using the traditional standard evaluation methods was different from the evaluation with GPI. Two parameters were found to have a significant impact on GPI: the $B G$ sampling frequency and the duration of algorithm application. A higher BG sampling frequency and a longer algorithm application duration resulted in an apparently better performance, as indicated by a lower GPI.

Conclusion The GPI is an alternative method for evaluating the performance of BG control algorithms. The blood glucose sampling frequency and the duration of algorithm application should be similar when comparing algorithms.

\section{Introduction}

Hyperglycemia and insulin resistance are common in critically ill patients (even those without diabetes mellitus [1-3]) and are associated with adverse outcome in a variety of clinical settings [4-7]. In two randomized controlled studies of mechanically ventilated patients admitted to a surgical and a medical intensive care unit (ICU), normalization of blood glucose (BG) (between 80 and $110 \mathrm{mg} / \mathrm{dl}$ ) with insulin significantly reduced morbidity and mortality rates $[8,9]$. BG control, aiming at normoglycemia, is now attempted in ICUs worldwide. This is usually performed by nurses or physicians who are instructed by 'manual' guidelines or algorithms [10-14]. These algorithms

$\mathrm{BG}=$ blood glucose; $\mathrm{GPI}=$ glycemic penalty index; $\mathrm{HGI}=$ hyperglycemic index; ICU: intensive care unit. 
are developed with the purpose of determining the insulin dose that is required to obtain normoglycemia based on intermittent BG readings. Computer-based protocols (as presented in [15-27]) have the potential to facilitate and improve glycemic control and to reduce the workload for medical staff. However, these 'new' protocols require stringent validation before they can replace the currently existing 'nurse-driven' insulin protocols.

Three types of methods exist for evaluating the adequacy of insulin titration algorithms. All of them, however, show weaknesses that may lead to erroneous conclusions. The first method simply computes the average of all BG readings. In spite of its popularity, it must be stressed that normoglycemia can be falsely assumed even in the presence of severely abnormal BG values. Indeed, hypoglycemic and hyperglycemic events can artificially lower or raise, respectively, the calculated average and can even balance each other, leading to an apparently 'normal' average BG.

A second method comprises single measurements; for example taking BG readings at a fixed time of day, the minimum/ maximum BG values, and the time needed to reach the target BG. Alternative single measurements count the number of hypoglycemic or hyperglycemic events. Although such measurements are useful, they do not capture the BG dynamics.

Recently, the hyperglycemic index (HGl) was presented as a third, more advanced, tool for assessing glucose control (in the ICU) with respect to hyperglycemic events [28]. The HGI is defined as the area under the glucose curve above 6.0 $\mathrm{mmol} / \mathrm{l}(108 \mathrm{mg} / \mathrm{dl})$ divided by the length of ICU stay. Two conditions to be satisfied before applying the HGl were proposed [29]. First, the number of BG measurements should be sufficiently high; ideally a near-continuous glucose read-out. Second, the considered sampling frequency should be comparable in both patient groups when comparing the adequacy of two insulin titration algorithms. It is important to note that area-under-the-curve methods (such as the HGI) currently rely on the assumed (linear) relationship between intermittent BG readings, since no reliable and accurate near-continuous glucose sensor is presently available $[15,19,30]$.

Another critical point of this technique is that outliers can potentially warp the obtained results due to the possible presence of extreme (hyperglycemic) observations that may have an impact on the computed area-under-the-curve. This is an important feature when realizing that sensor accuracy (and reliability) typically decreases as the BG level increases [3133]. It is clear that the presence of outliers also affect the computed average BG values (see Materials and methods). Finally, HGI only transforms the hyperglycemic, and not the hypoglycemic, glucose dynamics into a number. Of course, we acknowledge that the design of an alternative hypoglycemic index (as already suggested in [34]) would overcome this last aspect.

The aim of the current study was to design a tool for adequate comparison of BG control algorithms. In the first part of the study, we developed a grading system that scores normal, hypoglycemic, and hyperglycemic BG readings; the glycemic penalty index (GPI). In the second part of the study, the performance of the GPI was compared on an individual as well as a population basis with the current standard evaluation methods (average morning BG, mean of all BG readings and $\mathrm{HGI}$ ), using data from a selected set of patients. In the third part of the study, we investigated the importance or the weight of four clinically selected parameters (BG sampling frequency, duration of algorithm application, severity of disease, and type of illness) on GPI.

\section{Materials and methods}

\section{Mathematical computation of GPI}

We defined the GPI as a tool that scores BG readings in order to evaluate the overall BG dynamics obtained in the considered patient by applying a specific ICU insulin titration algorithm. The computation of the GPI used a penalty strategy that was based on clinical 'expert' knowledge. The glycemic target range in the ICU was defined as 80 to $110 \mathrm{mg} / \mathrm{dl}[8,9]$, with a penalty value for all $B G$ values lying in this range, therefore set at 0 . Hyperglycemic and hypoglycemic events were amplified (in terms of the assigned penalties) in relation to the magnitude of their deviation from the target range. Table 1 gives an overview of the glycemic threshold values that are generally accepted for use in the ICU. Each glycemic range was associated with a penalty $\rho$ leading to a staircase 'expert' penalty function when considering the full glycemic range (see Figure 1, dashed line).

We then smoothed this staircase function in order to avoid abrupt changes in the penalty function. However, the clinically accepted normoglycemic target range, the hypoglycemic alarm level (i.e., BG values below $40 \mathrm{mg} / \mathrm{dl}$ [35]), and the hyperglycemic alarm level (i.e., BG values above $200 \mathrm{mg} / \mathrm{dl}$ [8]) were respected in the design of the more smoothed function. This gave the advantage that penalties were gradually increasing as a function of the increasing deviation from the target range. Accordingly, BG measurement errors caused by sensor inaccuracies and methodology inaccuracies due to sampling handling only had a limited impact on the overall assessment of a BG algorithm.

The smooth penalty function was subsequently optimized by designing a polynomial function in the $B G$ ranges 20 to 79 $\mathrm{mg} / \mathrm{dl}$ and 111 to $250 \mathrm{mg} / \mathrm{dl}$. The squared differences between the staircase and the more smoothed function were minimized by applying ordinary least squares [36]. The penalty index corresponding to the normoglycemic range (80 to 110 $\mathrm{mg} / \mathrm{dl}$ ) was set at 0 . BG values lower than $20 \mathrm{mg} / \mathrm{dl}$ and higher 
Table 1

Penalty evaluation strategy showing threshold values and penalty values for the evaluation of blood glucose (BG) control in the intensive care unit (ICU)

\begin{tabular}{|c|c|c|c|c|}
\hline Range no. & Glycemic range (mg/dl) & Clinical description & Penalty $(\rho)$ & Reference \\
\hline 1 & $B G<40$ & Hypoglycemic alarm & 3 & [35] \\
\hline 2 & $40 \leq B G<60$ & Hypoglycemia & 2 & [35] \\
\hline 3 & $60 \leq B G<80$ & Slight hypoglycemia & 1 & [35] \\
\hline 4 & $80 \leq B G \leq 110$ & Normoglycemia & 0 & {$[8,9]$} \\
\hline 5 & $110<B G \leq 150$ & Slight hyperglycemia & 1 & [30] \\
\hline 6 & $150<B G \leq 200$ & Hyperglycemia & 2 & [30] \\
\hline 7 & $200<B G$ & Hyperglycemic alarm & 3 & [8] \\
\hline
\end{tabular}

than $250 \mathrm{mg} / \mathrm{dl}$ were assigned a maximum value to avoid that outliers would distort the obtained GPI (as can be the case with currently used evaluation methods; see above).

\section{Comparison of GPI with currently used evaluation methods}

The average morning BG, the average BG (i.e., the mean of all $B G$ readings), the $\mathrm{HGl}$, and the GPI were computed for each patient in the study set. Though the glycemic target range was 80 to $110 \mathrm{mg} / \mathrm{dl}$, we defined $120 \mathrm{mg} / \mathrm{dl}$ as a 'clinically acceptable' upper limit taking into account possible sensor inaccuraFigure 1

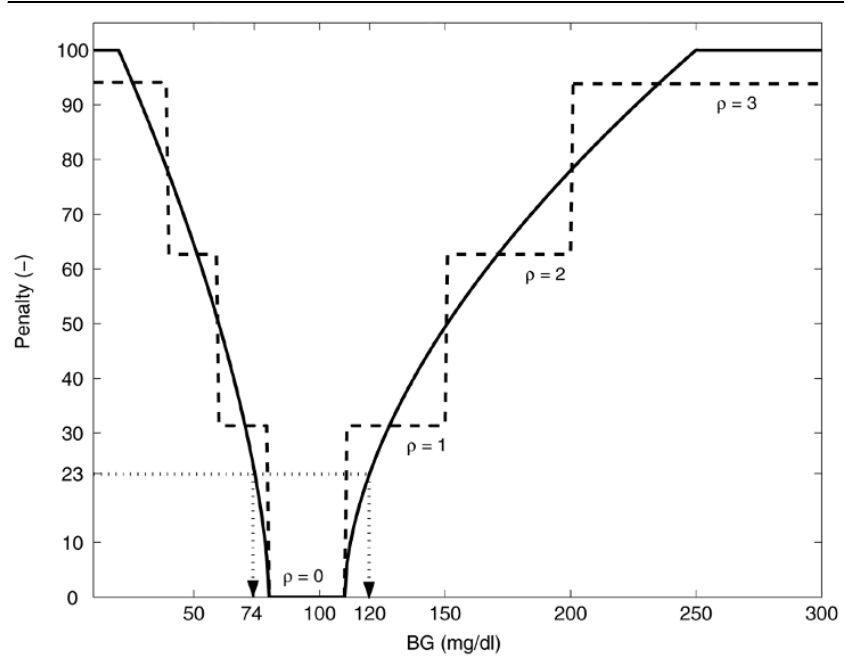

Penalty index as a function of blood glucose (BG). Each BG observation corresponds to a penalty. The dashed line represents the staircase penalty index function (here, the penalty is denoted as $\rho$ ). For reasons explained in the text, this staircase function was transformed into a more smoothed penalty index function, which is illustrated by the solid line. The penalties are symbolized by $\beta, \gamma$, and $\delta$ for the low, normal, and high BG measurements, respectively. The 'clinically acceptable' cut-off glycemic penalty index (GPI) equals 23 and corresponds to a 'clinically acceptable' BG range of 74-120 mg/dl. The target normoglycemic range, however, remains 80 to $110 \mathrm{mg} / \mathrm{dl}$ with a corresponding penalty value equal to 0 . cies and methodology inaccuracies due to sampling handling [37-41]. Therefore, the cut-off values for evaluating the performance of the BG algorithm were arbitrarily set as follows. Average morning BG readings below $120 \mathrm{mg} / \mathrm{dl}$, average BG values below $120 \mathrm{mg} / \mathrm{dl}$, and HGls below $12 \mathrm{mg} / \mathrm{dl}$ (i.e., an average hyperglycemic value below $108+12=120 \mathrm{mg} / \mathrm{dl}$ ) were labeled 'clinically acceptable'. The cut-off GPI that explained whether the insulin titration algorithm was acceptable or not was determined by entering $120 \mathrm{mg} / \mathrm{dl}$, as cut-off $B G$, to the developed smooth penalty function. Next, the performance of the existing standard evaluation methods and the GPI were compared both on individual and population basis.

\section{Study procedure and patient population}

We assembled two different data sets obtained from patients who had been admitted to the surgical ICU division of the University Hospital KU Leuven (Belgium) and who had been treated by the same nursing team but for whom a different BG sampling frequency was used. Whole BG in undiluted arterial blood was measured by means of the same glucose analyzer (ABL700 Radiometer Medical, Copenhagen) in both patient groups. The first patient group comprised 41 subjects (patient group 1) who were retrospectively selected from the data originally described in [8]. They were chosen to cover variable demographics (Table 2) and durations of stay in the ICU. The goal was to retrieve a representative sample for the larger patient group of [8] in terms of duration of intensive care and proportion of diagnostic subgroups. The BG sampling frequency and insulin titration guidelines (as described in [11]) were identical for all patients. The second patient group comprised 52 subjects (patient group 2) with variable demographics and duration of stay in the ICU, of whom only the first 2 days were considered, during which the sampling frequency was set at once every hour. Only those patients who were expected to have a duration of stay of more than 2 days were selected for this study. The titration was performed by the same nursing staff, who followed the same guidelines, as used for patient group 1. 
Table 2

Patient populations 1 and 2 (both coming from a surgical intensive care unit (ICU)) showing characteristics of both surgical ICU patient groups

\begin{tabular}{|c|c|c|}
\hline Variable & Patient group 1 & Patient group 2 \\
\hline No. of patients & 41 & 52 \\
\hline Male sex, no. (\%) & $27(65.8)$ & $29(55.8)$ \\
\hline Age, years (SD) & $59.8(17.6)$ & $65.2(16.1)$ \\
\hline Body mass index, kg/m² (SD) & $27.0(5.2)$ & $25.1(4.7)$ \\
\hline \multicolumn{3}{|l|}{ Reason for intensive care, no. (\%): } \\
\hline Cardiac surgery, type 1 & $11(26.8)$ & $32(61.5)$ \\
\hline Non-cardiac indication: & $30(73.2)$ & $20(38.5)$ \\
\hline Multiple trauma or severe burns, type 2 & $7(17.1)$ & $0(0)$ \\
\hline $\begin{array}{l}\text { Neurologic disease, cerebral trauma, or } \\
\text { complicated brain surgery, type } 3\end{array}$ & $4(9.8)$ & $2(3.9)$ \\
\hline $\begin{array}{l}\text { Complicated lung or esophageal thoracic } \\
\text { surgery, respiratory insufficiency, or both, type } \\
4\end{array}$ & $7(17.1)$ & $5(9.6)$ \\
\hline $\begin{array}{l}\text { Complicated abdominal surgery or peritonitis, } \\
\text { type } 5\end{array}$ & $5(12.2)$ & $10(19.2)$ \\
\hline Transplantation, type 6 & $3(7.3)$ & $2(3.9)$ \\
\hline Complicated vascular surgery, type 7 & $2(4.9)$ & $0(0)$ \\
\hline Other, type 8 & $2(4.9)$ & $1(1.9)$ \\
\hline APACHE II score (first 24 h) (SD) & $11(6)$ & $16(4)$ \\
\hline Mean $B G-m g / d l(S D)$ : & $108(37)$ & $104(29)$ \\
\hline Minimal BG, mg/dl & 37 & 37 \\
\hline Maximal BG, mg/dl & 379 & 307 \\
\hline
\end{tabular}

$\mathrm{SD}$, standard deviation.

Except for the different BG sampling frequency and the duration of algorithm application, both patient groups varied for type of illness and the APACHE II (Acute Physiology and Chronic Health Evaluation; [42]) score. The average APACHE II score was higher in group 2. The differences between the patient groups allowed us to analyze the influence (weight) of the four selected clinically relevant parameters (see below) on GPI in an appropriate way. Informed consent was obtained from the closest family member at ICU admission. The study protocol was approved by the Institutional Ethical Review Board.

\section{Definition of parameters}

Four different parameters were selected based on their clinically expected influence on GPI. The first parameter was the average $B G$ sampling frequency $(f)$, which was the average number of $B G$ readings (per time unit) that were available and used by the insulin titration algorithm. The conversion to time dimension was realized by taking the inverse of the frequency (e.g., $f=0.5 \mathrm{~h}^{-1}$ corresponds to a time interval of $2 \mathrm{~h}$ ). The second parameter was the duration of algorithm application $(D)$, which was the time period that the control algorithm was effectively used for a given patient. The next parameter was the severity of disease $(A)$, scored by the APACHE II score, still the most reported and used system in ICU and, therefore, the one selected for this study (although more recent scoring systems may perform better at grading severity of illness). The APACHE II score of the first $24 \mathrm{~h}$ after admission to the ICU was calculated for each patient using parameters of acute physiology and chronic healthcare. The final parameter under study was the type of illness. As an example, eight reasons for admission to the ICU were considered in this analysis: cardiac surgery (type 1), multiple trauma or severe burns (type 2), neurologic disease, cerebral trauma or complicated brain surgery (type 3), complicated lung or esophageal thoracic surgery, respiratory insufficiency, or both (type 4), complicated abdominal surgery or peritonitis (type 5), transplantation (type 6), complicated vascular surgery (type 7), and others (type 8).

\section{Statistics}

The Kruskal-Wallis test was used for comparing the medians of two or more groups of data. Depending on the distribution of the residuals, general and generalized linear models were built. In the general linear model, the Shapiro-Wilk test was 
applied for testing the normality of the residuals. The determination of the significance (weight) of the specific parameter on GPI (i.e., 'input selection' for the model) was based on F-tests for the general linear model and the likelihood ratio Chi-square statistics for the generalized linear model. For the last type of model, Wald statistics were used. Pearson's correlation coefficients $(R)$ were calculated for quantifying the relation between continuous variables. In all applied tests $p$ values $<$ 0.05 were considered to be significant.

\section{Results}

\section{Mathematical computation of GPI}

The clinically defined staircase penalty function was transformed to a more smoothed penalty function. The obtained function was mathematically formulated as follows:

For time step $t=1$ to $N_{\text {total }}$ :

$B G_{t}<20 \mathrm{mg} / \mathrm{dl}: \beta_{i}=100$

$20 \mathrm{mg} / \mathrm{dl} \leq B G_{t}<80 \mathrm{mg} / \mathrm{dl}: \beta_{i}=7.4680\left(80-B G_{t}\right)^{0.6337}$,

$80 \mathrm{mg} / \mathrm{dl} \leq B G_{t} \leq 110 \mathrm{mg} / \mathrm{dl}: \gamma_{j}=0$,

$110 \mathrm{mg} / \mathrm{dl}<B G_{t} \leq 250 \mathrm{mg} / \mathrm{dl}: \delta_{k}=6.1767\left(B G_{t}-110\right)^{0.5635}$,

$250 \mathrm{mg} / \mathrm{dl}<B G_{t}: \delta_{k}=100$

where $\beta_{i}$ was the penalty index for a glucose reading $B G_{t}$ of the hypoglycemic range (i.e., $B G_{t}<80 \mathrm{mg} / \mathrm{dl}$ ), $\gamma_{j}$ for the normoglycemic range (i.e., $80 \mathrm{mg} / \mathrm{dl} \leq B G_{t} \leq 110 \mathrm{mg} / \mathrm{dl}$ ), and $\delta_{k}$ for the hyperglycemic range (i.e., $B G_{t}>110 \mathrm{mg} / \mathrm{dl}$ ). The indices $i, j$, and $k$ were used to count the number of hypoglycemic, normoglycemic, and hyperglycemic events, respectively. The symbol that represents the number of BG measurements in the full glycemic range (that were available for the considered patient) was $N_{\text {total. }}$ This more smoothed function is illustrated in Figure 1 (solid line).

All BG values from a patient corresponded to specific penalty values as directly followed from the smoothed function. Next, the GPI was calculated for each patient:

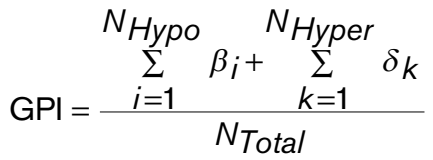

where $N_{\text {Hypo }}$ is the symbol for the number of BG measurements in the hypoglycemic range, and $N_{\text {Hyper }}$ the symbol for the hyperglycemic range. The relative contribution of the hypoglycemic values to GPI (denoted as $C_{\text {Hypo }}$ ) was determined as follows:

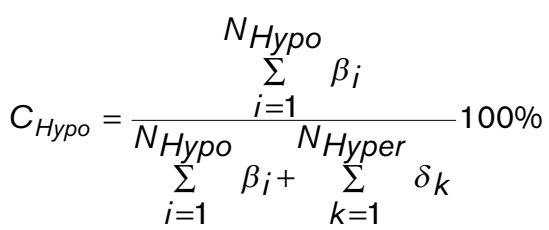

Analogously, the relative contribution of the hyperglycemic values to GPI (denoted as $C_{\text {Hyper }}$ ) was computed as follows:

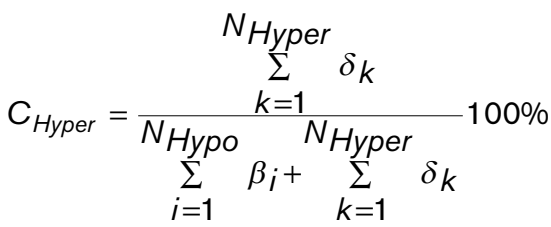

\section{Comparison of GPI with currently used methods}

The 'clinically acceptable' upper limit BG $(120 \mathrm{mg} / \mathrm{dl})$ was entered into the above developed smoothed penalty function, giving 23 as 'clinically acceptable' cut-off GPI. Next, the inverse smoothed penalty function was used to compute the lower limit BG that corresponded to $\mathrm{GPI}=23$ (Figure 1). The 'clinically acceptable' BG range was found to be 74 to 120 $\mathrm{mg} / \mathrm{dl}$ but the glycemic target range remained at 80 to 110 $\mathrm{mg} / \mathrm{dl}$. In other words, a computed GPI below 23 allowed us to conclude that the insulin titration algorithm was able to control BG according to the clinical requirements. Ideally, however, all BG readings should fall within the 80 to $110 \mathrm{mg} / \mathrm{dl}$ zone leading to a GPI equal to 0 .

Table 3 gives a detailed overview of the results of the evaluation methods (average morning BG, average BG, HGI, GPI, and the relative contribution of the low $\left(C_{\text {hypo }}\right)$ and high $\left(C_{\text {hyper }}\right)$ $\mathrm{BG}$ observations to the computed $\mathrm{GPI}$ ) that are applied to patient group 1. A summary of this is presented in Table 4. Figures 2 and 3 further summarize the performance differences between the evaluation methods applied to the individual patients. The correlation coefficients for the already existing measures with respect to GPI are depicted in each respective panel. Finally, Figure 4 illustrates the BG profile of patient no. 19 (top panel, no misleading effect of standard assessment methods), whose BG was tightly controlled, and patient no. 11 (bottom panel, assessment misled by average (morning) BG and $\mathrm{HGl}$ ) with poor BG control (see also Table 4 for exactly computed measures).

In most studies, however, the BG control algorithm is evaluated using the patient population rather than on individual patients $[8,9,20,21,27]$. The population results for patient group 1 are mentioned in Table 4 . The most appropriate way to present the population $\mathrm{HGl}$ and GPI values is by calculating the median and $25 \%$ to $75 \%$ interquartile (IQ) range as these data were not normally distributed. 
Critical Care Vol 12 No 1 Van Herpe et al.

Table 3

Blood glucose (BG) control assesment; evaluation of BG control by computing the average morning BG, the average BG, the hyperglycemic index (HGI), and the glycemic penalty index (GPI) for patient group 1

\begin{tabular}{|c|c|c|c|c|}
\hline Patient no. & $\begin{array}{l}\text { Average morning BG } \\
(\mathrm{mg} / \mathrm{dl})\end{array}$ & Average BG (mg/dl) & HGI (mg/dl) & $\begin{array}{l}\text { GPI }\left(C_{\text {Hypo }}(\%)-\right. \\
\left.C_{\text {Hyper }}(\%)\right)\end{array}$ \\
\hline 1 & 161 & 143 & 39 & $49(16.2-83.8)$ \\
\hline 2 & 129 & 123 & 17 & $27(4.0-96.0)$ \\
\hline 3 & 124 & 141 & 28 & $42(9.6-90.4)$ \\
\hline 4 & 165 & 131 & 41 & $45(23.0-77.0)$ \\
\hline 5 & 97 & 101 & 6 & $20(34.7-65.3)$ \\
\hline 6 & 77 & 105 & 9 & $22(36.6-63.4)$ \\
\hline 7 & 104 & 115 & 9 & $18(4.2-95.8)$ \\
\hline 8 & 129 & 127 & 27 & $35(18.4-81.6)$ \\
\hline 9 & 93 & 132 & 26 & $37(29.5-70.5)$ \\
\hline 10 & 109 & 106 & 8 & $16(23.5-76.5)$ \\
\hline 11 & 103 & 100 & 4 & $27(49.8-50.2)$ \\
\hline 12 & 97 & 117 & 15 & $29(21.4-78.6)$ \\
\hline 13 & 100 & 101 & 4 & $10(31.2-68.8)$ \\
\hline 14 & 103 & 113 & 9 & $18(11.5-88.5)$ \\
\hline 15 & 98 & 98 & 4 & $13(47.5-52.5)$ \\
\hline 16 & 111 & 114 & 18 & $28(27.5-72.5)$ \\
\hline 17 & 101 & 101 & 6 & $15(40.0-60.0)$ \\
\hline 18 & 104 & 105 & 4 & $7(1.7-98.3)$ \\
\hline 19 & 97 & 99 & 1 & $5(39.0-61.0)$ \\
\hline 20 & 102 & 99 & 3 & $9(25.7-74.3)$ \\
\hline 21 & 102 & 107 & 4 & $12(16.2-83.8)$ \\
\hline 22 & 126 & 115 & 10 & $17(11.9-88.1)$ \\
\hline 23 & 60 & 101 & 21 & $56(59.7-40.3)$ \\
\hline 24 & 102 & 135 & 19 & $23(0-100)$ \\
\hline 25 & 101 & 107 & 7 & $9(10.3-89.7)$ \\
\hline 26 & 100 & 106 & 8 & $19(29.7-70.3)$ \\
\hline 27 & 112 & 107 & 6 & $14(27.8-72.2)$ \\
\hline 28 & 104 & 111 & 10 & $15(1.3-98.7)$ \\
\hline 29 & 105 & 110 & 7 & $10(0-100)$ \\
\hline 30 & 120 & 177 & 62 & $61(5.2-94.8)$ \\
\hline 31 & 110 & 96 & 6 & $23(57.1-42.9)$ \\
\hline 32 & 99 & 102 & 5 & $11(18.3-81.7)$ \\
\hline 33 & 120 & 119 & 14 & $21(12.1-87.9)$ \\
\hline 34 & 96 & 96 & 2 & $9(51.2-48.8)$ \\
\hline 35 & 94 & 97 & 3 & $12(48.0-52.0)$ \\
\hline 36 & 157 & 194 & 74 & $55(19.5-80.5)$ \\
\hline 37 & 94 & 97 & 4 & $13(49.1-50.9)$ \\
\hline
\end{tabular}




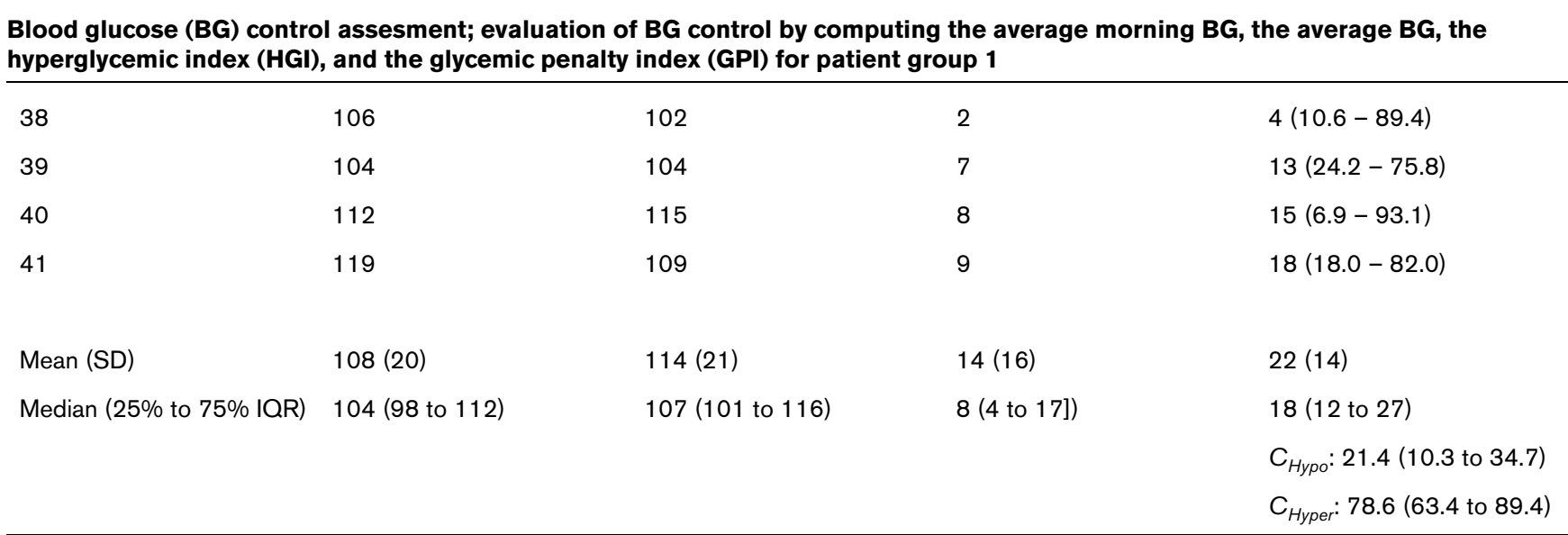

$\mathrm{IQR}$, interquartile range; SD, standard deviation.

\section{Weight determination for the selected parameters}

The impact of the variables under study on GPI for the patients belonging to patient group 2 and 1 (but considering only the data of the first $48 \mathrm{~h}$ in the latter) is illustrated in Figure 5. As the duration of algorithm application was set at $48 \mathrm{~h}$, this variable was not included in this analysis. The $p$ values of the null hypothesis that the GPI medians per group are equal are noted in each respective panel (only significant inequality for the average BG sampling frequency). Multiple regression analysis on these data revealed that the average BG sampling frequency was the only parameter that significantly $(p=0.0051)$ impacted the assessment of insulin titration algorithms: an inversely proportional effect was observed.

Figure 6 illustrates the independent impact of all four variables under study on GPI for patient group 1 (considering all the available data of this group). The obtained $p$ values of the null hypothesis that the GPI medians per group are equal are again noted in the respective panels (significant inequality for the average BG sampling frequency and duration of algorithm application). Multiple regression analysis returned that both duration of algorithm application $(p=0.032)$ and the product of duration of algorithm application and average BG sampling frequency ( $p=0.025$ ) significantly influenced GPI. The first parameter was directly proportional to GPI whereas the product was inversely proportional to GPI. Moreover, a negative correlation ( $R=-0.42, \mathrm{p}=0.0069$ for the 'no-correlation' null hypothesis) between the variables duration of algorithm application and average BG sampling frequency was found.

The impact of the duration of algorithm application on GPI is further clarified in Figure 7 for the patients (from group 1) who stayed for at least $100 \mathrm{~h}$ in the ICU. Every $24 \mathrm{~h}$, the GPI was computed based on all previous BG observations of each par-

Table 4

Blood glucose (BG) control assessment for a subset of patient group 1 (summary of Table 3); evaluation of BG control by computing the average morning BG, the average BG, the hyperglycemic index (HGI), and the glycemic penalty index (GPI) for a subset of patient group 1

\begin{tabular}{|c|c|c|c|c|}
\hline Patient no. & $\begin{array}{l}\text { Average morning BG } \\
(\mathrm{mg} / \mathrm{dl})\end{array}$ & Average BG $(\mathrm{mg} / \mathrm{dl})$ & HGI (mg/dl) & $\underset{(\%))}{\operatorname{GPI}}\left(C_{\text {Hypo }}(\%)-C_{\text {Hyper }}\right.$ \\
\hline 1 & 161 & 143 & 39 & $49(16.2-83.8)$ \\
\hline 11 & 103 & 100 & 4 & $27(49.8-50.2)$ \\
\hline 19 & 97 & 99 & 1 & $5(39.0-61.0)$ \\
\hline Mean (SD) & $108(20)$ & $114(21)$ & $14(16)$ & $22(14)$ \\
\hline \multirow[t]{3}{*}{ Median (25\% to $75 \%$ IQR) } & 104 (98 to 112$)$ & 107 (101 to 116$)$ & 8 (4 to 17$)$ & 18 (12 to 27$)$ \\
\hline & & & & $C_{\text {Hypo: }}: 21.4$ (10.3 to 34.7$)$ \\
\hline & & & & $C_{\text {Hyper }}: 78.6(63.4$ to 89.4$)$ \\
\hline
\end{tabular}



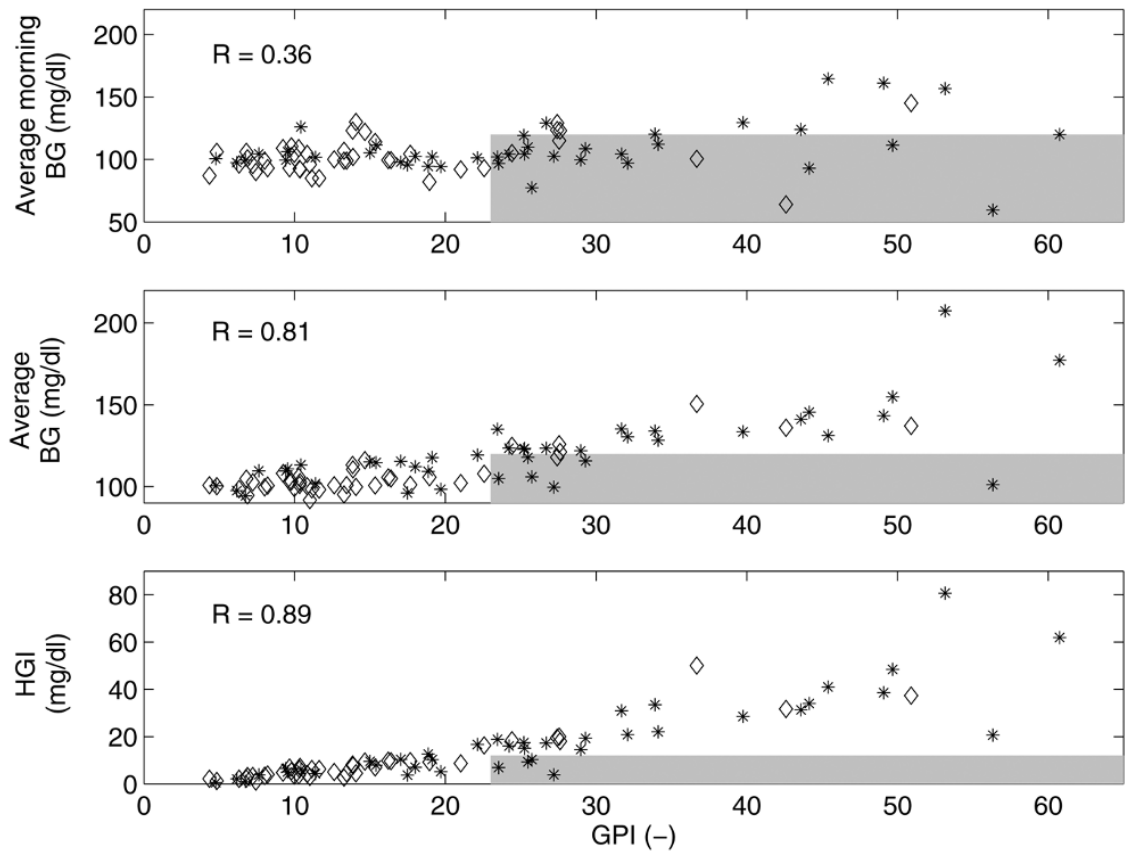

Standard evaluation techniques versus glycemic penalty index (GPI) for patient group 2 and 1 (but considering only the data of the first $48 \mathrm{~h}$ in the latter). The results of the standard evaluation methods are plotted against the results of the new proposed assessment tool (GPI). The top panel shows the average morning blood glucose (BG) readings as a function of GPI. The middle panel represents the average BG values versus the GPI values. Finally, the bottom panel illustrates the computed HGI values as a function of GPI. The shaded area contains those patients whose BG profile was evaluated differently: 'clinically acceptable' for the standard measures, 'clinically unacceptable' for GPI. The stars denote the patients from group 1 whereas the diamonds represent the patients from group 2. The correlation coefficients $(R)$ for the existing measures with respect to GPI are depicted in the respective panels.

ticular patient. Each line of Figure 7 represents the GPI evolution of this specific patient as a function of the number of data (i.e., the time spent in the ICU) that were considered in the calculation process of GPI. For the majority of the patients, a decreasing GPI trend could be observed as more data (longer duration of the applied algorithm) were taken into consideration.z

\section{Discussion}

In this study we developed the GPI as a tool for assessing the dynamics of glycemic control in ICU patients. The designed formula returned a number between 0 and 100 with an 'ideal' level of $O$ (indicating that all measured BG values fell within the normoglycemic target range) and a 'clinically acceptable' level of 23. Further, it was shown that GPI summarized the monitored glucose profile into one number more precisely than the traditional evaluation tools based on currently available clinical expertise. Finally, the average BG sampling frequency and the duration of algorithm application were found to be parameters that should be comparable for patient groups when comparing the performance of insulin titration algorithms.

\section{Mathematical computation of GPI}

The developed GPI tool summarizes the level of tight glycemic control into a single number based on a grading system that scores low and high BG readings depending on their deviation from the target range. There are many advantages of GPI over the current standard methods. First, since both low and high $B G$ readings are taken into account, GPI measures the overall BG dynamics. Since the assigned penalties are always absolute (positive), it is not possible that hypoglycemic and hyperglycemic penalties balance each other as can be the case when computing the average (morning) BG.

Second, only the BG readings that were effectively monitored are used in the GPI evaluation tool. Accordingly, unlike for area-under-the-curve methods, the GPI does not rely on any assumed (linear) relationship between measurements. While awaiting the creation of reliable near-continuous sensor devices for BG monitoring in an ICU setting, this is an important aspect as these assumed (linear) relations between observations do not necessarily approach the real (non-linear) blood glucose dynamics.

Third, a smooth penalty function (Figure 1) forms the basis of GPI leading to a gradual increase of the assigned penalties as the deviations from normoglycemia are enlarging. Measurement errors caused by sensor inaccuracies and methodology inaccuracies due to sampling handling have only a 

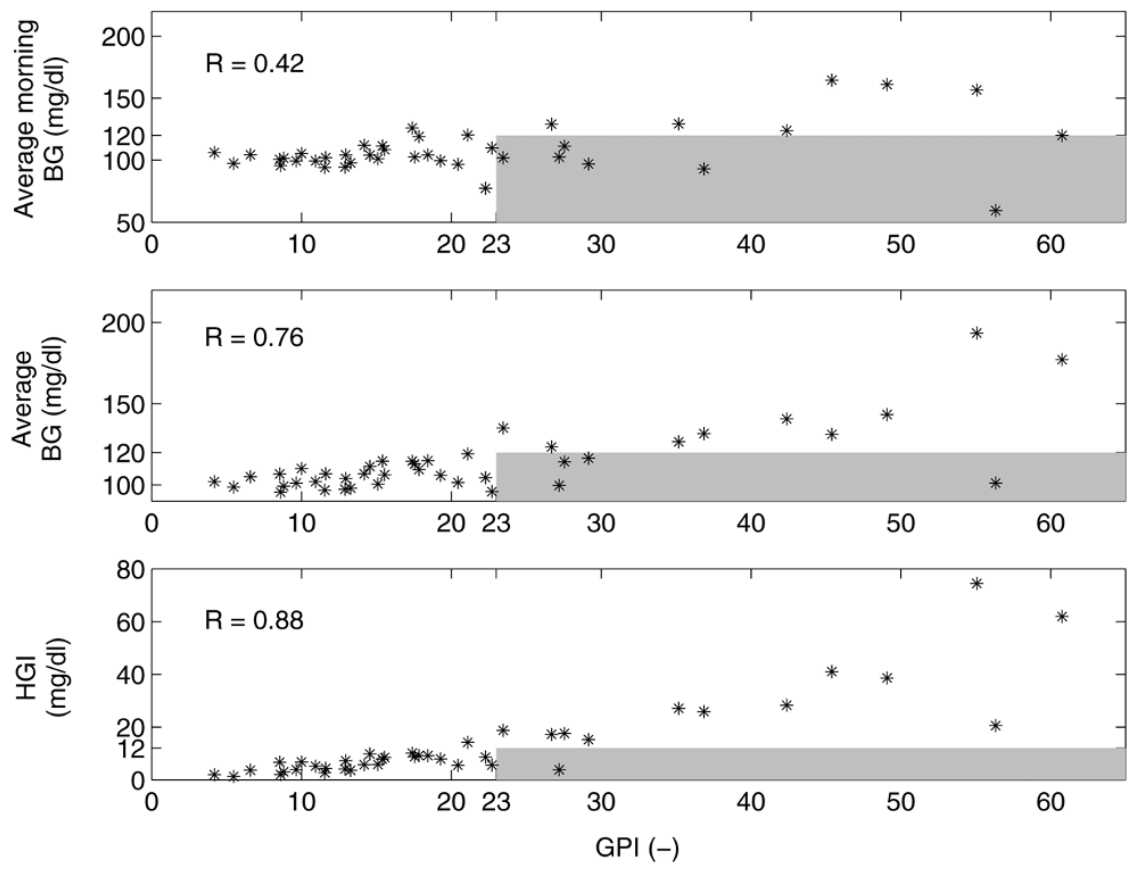

Standard evaluation techniques versus glycemic penalty index (GPI) for patient group 1 (considering the full dataset). The blood glucose (BG) profiles of the patients of group 1 are evaluated by applying the same standard techniques as mentioned in Figure 2 and are again presented as a function of GPI. The patients that belong to the shaded area got a different algorithm evaluation dependent on the method that was used (respective 'standard' method versus GPI). The shaded area contains those patients whose BG profile was evaluated differently: 'clinically acceptable' for the standard measures, 'clinically unacceptable' for GPI.

small level of influence on the assignment of the penalty, accordingly.

A fourth important feature of GPI is the independency of outlier measurements. Due to the imposed limits in the penalty function (if $B G<20 \mathrm{mg} / \mathrm{dl}$ or $B G>250 \mathrm{mg} / \mathrm{dl}$, then $\beta=\delta=100$ ), extreme BG measurements (that may be related to sensor/ methodology inaccuracies) cannot mislead the general algorithm assessment. Moreover, BG values lower or higher than these imposed limits would not lead to a clinically different treatment. This concept formed the basis of the specific region strategy in the error grid analysis for the evaluation of glucose sensors, as reported previously $[32,33,43]$.

Finally, the computation of the relative contribution of the hypoand hyperglycemic events to GPI allows us to further interpret the obtained GPI value. If we consider the BG profile of patient no. 1 (from patient group 1) as an example (see Table 4), based on the high GPI that was obtained (GPI $=49>23$ ) it could be concluded that BG was poorly controlled in this patient. The relative contributions (expressed in terms of percentage) of the hypo- and hyperglycemic events $\left(C_{H y p o}\right.$ and $C_{\text {Hyper }}$, respectively) to GPI informed the clinician whether this non-optimal control behavior was caused by particularly low glucose events (if $\mathrm{C}_{\text {Hypo }}>75 \%$ ), high glucose events (if $\mathrm{C}_{\text {Hyper }}$ $>75 \%$ ), or both (if $C_{\text {Hypo }} \approx C_{\text {Hyper }}$ ). The non-optimal performance of the algorithm for this patient example was mainly caused by the hyperglycemic events due to the large value for $\mathrm{C}_{\text {hyper }}$ (see Table 4).

\section{Comparison of GPI with currently used methods}

The computed GPI can be used to appropriately evaluate the level of tight glycemic control in a single patient based on clinical expertise. Existing methods may mislead an assessment, as is shown for patient no. 11 in Figure 4 (bottom panel). The average morning BG $(103 \mathrm{mg} / \mathrm{dl} \leq 120 \mathrm{mg} / \mathrm{dl})$, the average $B G(100 \mathrm{mg} / \mathrm{dl} \leq 120 \mathrm{mg} / \mathrm{dl})$, and the HGl $(4 \mathrm{mg} / \mathrm{dl} \leq 12 \mathrm{mg} /$ dl) all suggest strict glycemic control whereas the GPI (27> 23) denotes the less tightly controlled BG signal based on clinical expert knowledge. Both hypoglycemic and hyperglycemic events can be observed in the BG profile, which is further confirmed by the similarity between $C_{\text {Hypo }}$ and $C_{\text {Hyper }}$ for this patient.

Figures 2 and 3 summarize the assessment of the individual BG profiles by applying the existing methods and GPI. The shaded area is defined by the GPI 'clinical unacceptability' cutoff $(G P I>23)$ and the 'clinical acceptability' limits of the known techniques (average (morning) BG $\leq 120 \mathrm{mg} / \mathrm{dl}, \mathrm{HGl} \leq$ $12 \mathrm{mg} / \mathrm{dl}$ ). In other words, the evaluation of the BG profiles of the patients belonging to this area may be misled by the existing methods (particularly the average morning $B G$ and average $B G$, due to their high number of patients in the shaded areas and, to a lesser degree, the HGI). In fact, only few BG 

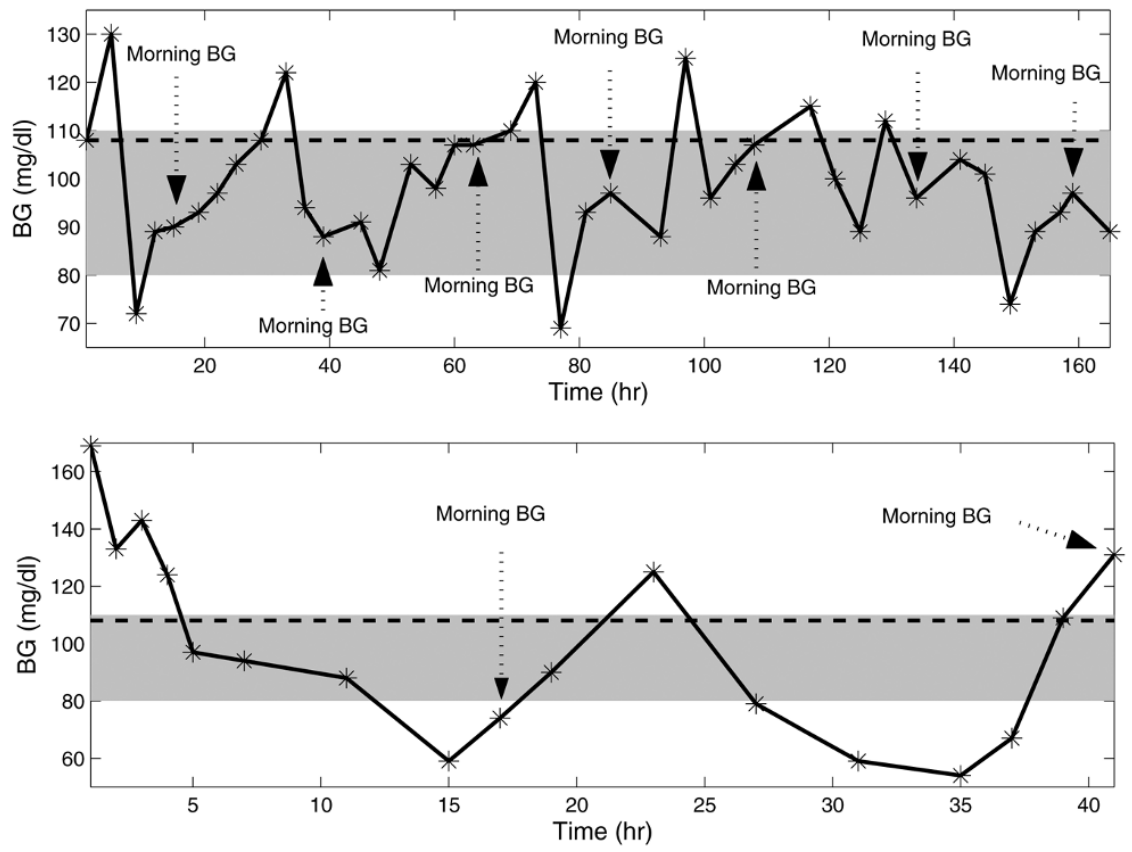

Measured blood glucose (BG) signals of patient no. 19 and 11. The measured BG readings (ABL700 Radiometer Medical) of patient no. 19 (top panel) and 11 (bottom panel) from group 1 are represented by stars. These BG profile examples denote tight glycemic control for patient no. 19 but rather poor glycemic control for patient no. 11. It is important to note that different time scales were used as patient no. 11 stayed in the intensive care unit (ICU) for only a short time period. Further, the obtained BG measurements were linearly interpolated. The normal BG range (target range) is indicated by the shaded area $(80-110 \mathrm{mg} / \mathrm{dl})$. The hyperglycemic index $(\mathrm{HGl})$ is the area under the glucose curve above $6 \mathrm{mmol} / \mathrm{l}(108 \mathrm{mg} / \mathrm{dl}$, as illustrated by the dashed line [28]). The morning BG values are indicated by the dotted arrows. Table 4 shows all computed measures in detail. Based on clinical expert knowledge, it can be observed that the assessment of the BG profile of patient no. 11 using the glycemic penalty index (GPI) is different from the evaluation with average (morning) BG and HGI.

profiles were evaluated differently with $\mathrm{HGl}$ indicating that this method most approaches the clinical 'expert' GPI function. This also occurs in the high correlation coefficients for HGI and GPI.

The assessment of the performance of the BG algorithm on a population basis also depended on the selected technique. As observed in Table 4, the average morning BG $(108 \pm 20 \mathrm{mg} /$ $\mathrm{dl})$ and the average BG (114 $\pm 21 \mathrm{mg} / \mathrm{dl})$ were below $120 \mathrm{mg} /$ $\mathrm{dl}$ suggesting that the algorithm under study was adequate. The computed IQ ranges for the average (morning) BG, both below $120 \mathrm{mg} / \mathrm{dl}$, confirmed this hypothesis. The computed $\mathrm{IQ}$ ranges of $\mathrm{HGI}$ and $\mathrm{GPI}$, however, indicated that the applied algorithm did not result in clinically acceptable BG control for at least $25 \%$ of the patients. Indeed, a quarter of the HGI values were above $17 \mathrm{mg} / \mathrm{dl}(>12 \mathrm{mg} / \mathrm{dl})$ and a quarter of the GPI values were above 27 (>23).

This study shows that the two most traditional measures (average morning BG and average BG) used for summarizing the overall glycemic control behavior, can mislead assessments of $B G$ algorithms. This confirms the results described in [44]. Techniques that take into account the duration of hyperglycemia/hypoglycemia, such as $\mathrm{HGl}$ or the recently proposed 'notional duration of hyperglycemia/hypoglycemia' (i.e., the time since the observation of an abnormal BG till it returns to the accepted range [44]) may better indicate tight glycemic control. The GPI technique proposed in this manuscript, however, is explicitly founded on ICU expertise and may therefore be an alternative (or at least a supplemental) tool for adequately evaluating insulin titration algorithms in the ICU. The BG profiles of most individual patients were equally assessed using GPI and $\mathrm{HGl}$, except for some patients as was illustrated in Figures 2 and 3 (shaded areas of the bottom panels).

A first weakness of the GPI is the non-consideration of the duration of hypoglycemic and hyperglycemic episodes since no (linear) relationship between discrete-time BG observations is assumed. Accordingly, the algorithm assessment may be misleading, as the number of intermittent BG measurements (and the number of assigned penalties) can typically be higher with unstable BG behavior (i.e., BG observations outside the normoglycemic target range). Only areaunder-the-curve measures (like $\mathrm{HGl}$ ) can potentially take into consideration the duration of these glycemic deviations under the assumption that the imposed (linear) relationship between the measurements approaches the real blood glucose dynamics. Moreover, the duration of deviating episodes can only be precisely taken into account with a reliable and accurate nearcontinuous glucose sensor. The use of such a device for the 

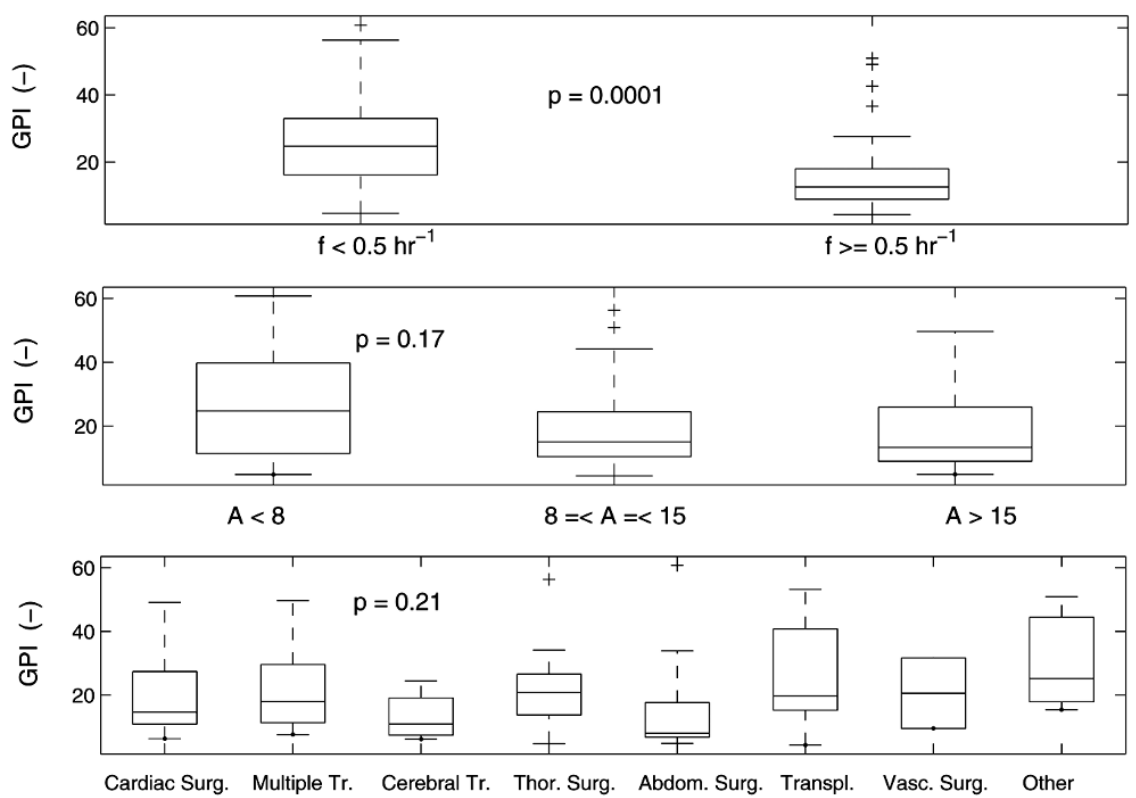

Univariate relationship between patient groups 2 and 1 (but considering only the data of the first $48 \mathrm{~h}$ in the latter). Univariate relationship (expressed in box plots) between average BG sampling frequency ( $f$ ) and GPI (top panel), APACHE II score $(A)$ and glycemic penalty index (GPI) (middle panel), and type of illness and GPI (bottom panel) for patient group 2 and 1 (but considering only the data of the first $48 \mathrm{~h}$ in the latter). The $\mathrm{p}$ values of the null hypothesis that the GPI medians per group are equal are mentioned in each panel. A significant difference was found for the average blood glucose (BG) sampling frequency: the BG profiles with $f \geq 0.5 \mathrm{~h}^{-1}$ (i.e., time intervals less than $2 \mathrm{~h}$ ) are related to stricter glycemic control (lower GPI).

evaluation of a BG algorithm even allows us to label the GPI measure as area-under-the-curve method (that incorporates the duration of glycemic deviations), since then a penalty is 'continuously' assigned to each BG (measured at each time instant, e.g., every minute) and since hypoglycemic and hyperglycemic deviations cannot balance each other. While awaiting reliable near-continuous glucose sensors [15,19,45], it is advised to sample BG at fixed time intervals (e.g., every hour or every $2 \mathrm{~h}$ for the duration of the study) to minimize the effect of the current weakness.

A second weakness of GPI is the ignorance of the severity of extreme (but exceptional) BG measurements due to hypo- and hyperglycemic cut-off values (e.g., $\delta_{300 \mathrm{mg} / \mathrm{dl}}=\delta_{450 \mathrm{mg} / \mathrm{dl}}=100$ ). Though the reasons for using these cut-off values are well founded (see above), we advise counting the number of alarm BG observations (i.e., BG $<40 \mathrm{mg} / \mathrm{dl}$ [35] and BG $>200 \mathrm{mg} /$ dl [8]) to better interpret the obtained GPI.

Previous studies have already indicated the relationship between improved clinical outcomes on the one hand and reduced average morning $B G[8,9]$ and reduced $\mathrm{HGI}[28]$ on the other. It is important to note that the relationship between GPI and clinical outcome has not been shown yet. The design of GPI is purely founded on currently available clinical expertise. Future studies are necessary to verify whether low GPIs effectively correspond to reduced mortality and morbidity, which is however expected from a clinical 'expert' point of view and from the high correlation between GPI and HGI.

\section{Weight determination for the selected parameters}

The BG sampling frequency in the insulin titration guidelines used in patient group 1, varied as a function of the level of glycemic control. When the blood glucose was more difficult to control (unstable glucose dynamics), more frequent sampling occurred. The full patient data of group 1 comprised the initial (unstable) and more chronic (stable) phase of each patient's stay in the ICU. An increasing duration of algorithm application (which implicitly indicates a longer stay in the ICU, typically associated with more stable glucose dynamics) artificially improved the average overall BG control behavior leading to lower GPls (see second panel of Figure 6). Figure 7 additionally clarifies the relationship between GPI and duration of algorithm application. The GPI decreases when more data (i.e., longer time/duration in the ICU) are considered in its computation process.

Further, this increasing duration of algorithm application lowered the average BG sampling frequency (expressed in the negative correlation between duration of algorithm application and average BG sampling frequency) since less BG observations were required in the chronic 'stable' period (due to the nature of the used protocol). Therefore, the first panel of Figure 6 that illustrates the relationship between tight glycemic con- 

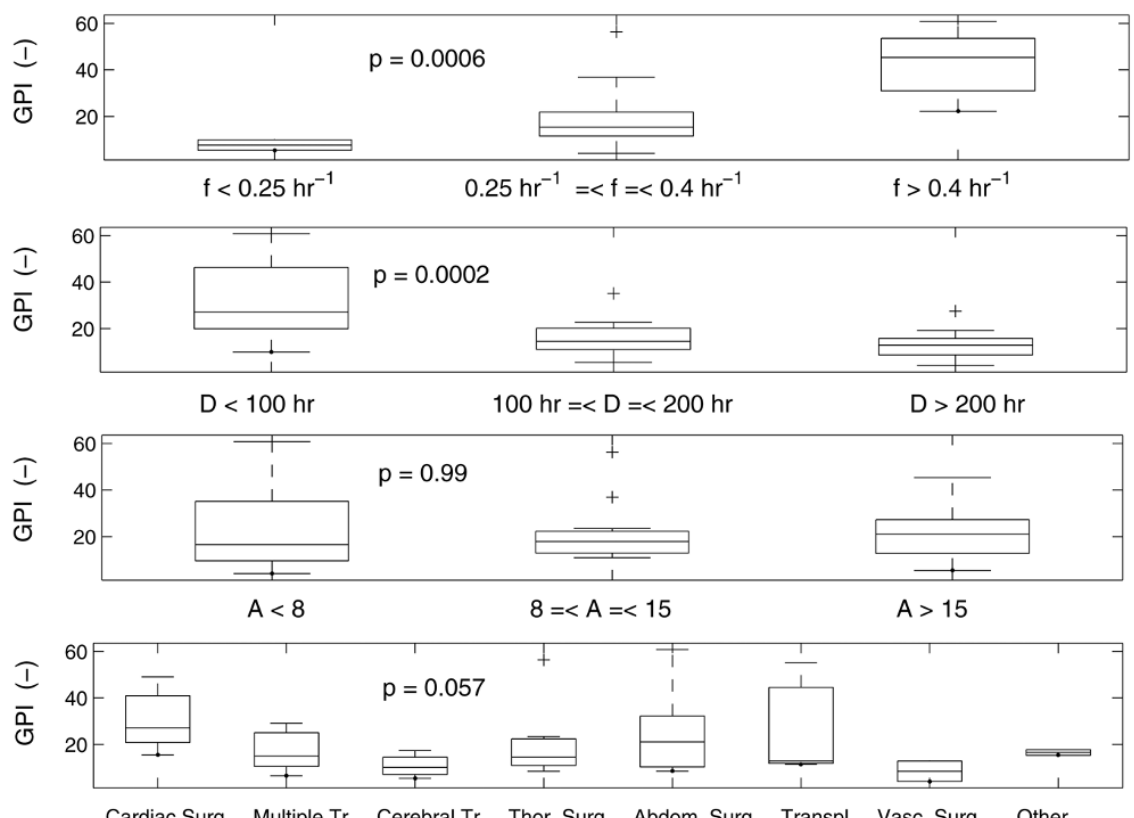

Univariate relationships in patient group 1. Univariate relationship (expressed in box plots) between average blood glucose (BG) sampling frequency $(f)$ and glycemic penalty index (GPI) (top panel), duration of algorithm application (D) and GPI (second panel), APACHE II score (A) and GPI (third panel), and type of illness and GPI (bottom panel) based on patient group 1. The $p$ values of the null hypothesis that the GPI medians per group are equal are mentioned in each panel. A significant difference was found for the average BG sampling frequency and the duration of algorithm application. The longer the algorithm is applied to the patient and the longer the time intervals between successive measurements, the tighter the glycemic control (lower GPI). The apparently contradictory impact of the BG sampling frequency on GPI can be explained by the negative correlation between the variables duration of algorithm application and average BG sampling frequency (see text).

\section{Figure 7}

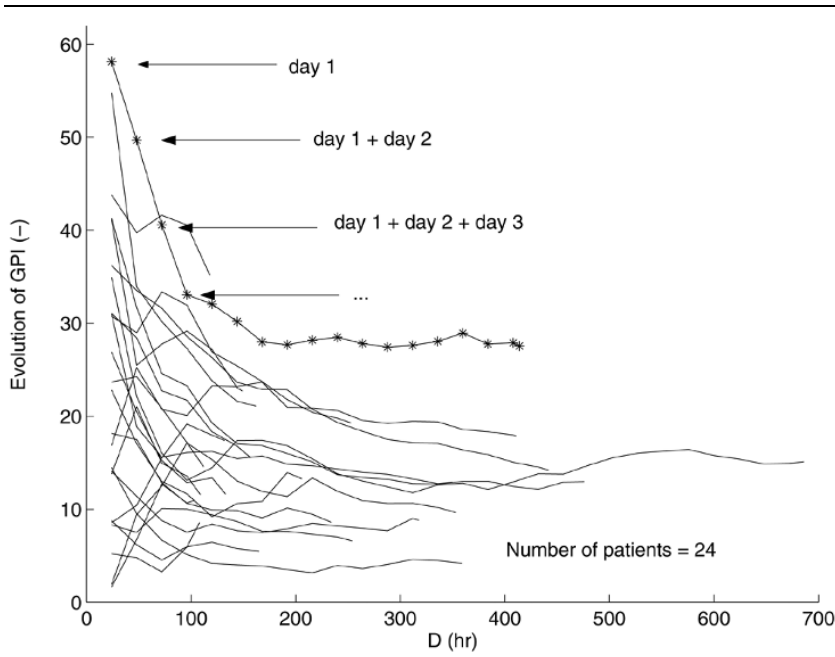

Evolution of the glycemic penalty index (GPI) as a function of duration of algorithm application. The evolution of the GPI values as a function of duration of algorithm application $(D)$ for the patients of group 1 who stayed for at least $100 \mathrm{~h}$ in the intensive care unit (ICU). Each line represents a patient. For example, the first star represents the GPI value that is calculated based on the blood glucose (BG) observations of the first $24 \mathrm{~h}$ of that specific patient. The second star gives the GPI value based on the measured BG signal of the first $48 \mathrm{~h}$; the third GPI value is computed based on the data of the first $72 \mathrm{~h}$; etc. For the majority of the patients, a decreasing GPI trend was observed. trol (low GPI) and a low average BG sampling frequency is explained by the long time that the algorithm was applied to the patients of group 1. In case the duration of algorithm application was kept constant and limited (only the first $48 \mathrm{~h}$ after admission), an increase of the BG sampling frequency resulted in more strict BG control (lower GPI) as depicted in the top panel of Figure 5. It can be concluded that both duration of algorithm application and average BG sampling frequency are two important parameters that should be taken into consideration when assessing or comparing different BG control algorithms.

\section{Practical use}

For the design of future studies that compare the performance of different insulin titration algorithms applied to critically ill patients, we encourage other research groups to rely on the 'similarity' condition: the duration of algorithm application and the BG sampling frequency should be similar in patient groups. We further encourage other groups to consider GPI as supplemental tool to other advanced measures (e.g., HGI, 'notional duration of hyperglycemia/hypoglycemia') besides more traditional measures (e.g., average morning BG, average $\mathrm{BG}$ ) for adequately assessing the overall level of (BG) control. 


\section{Conclusion}

The use of nurse-driven BG control algorithms is becoming standard practice in ICUs. New (semi-automated) insulin titration algorithms are currently under development but require an appropriate evaluation before accepting them as state-of-theart. In this study, we presented the computation of the GPI as a tool to compare different BG control algorithms. This index encompasses the overall BG dynamic behavior per patient in a single number based on clinical expertise. The method is affected by BG sampling frequency and duration of algorithm application, which should be similar for adequate comparison of these algorithms.

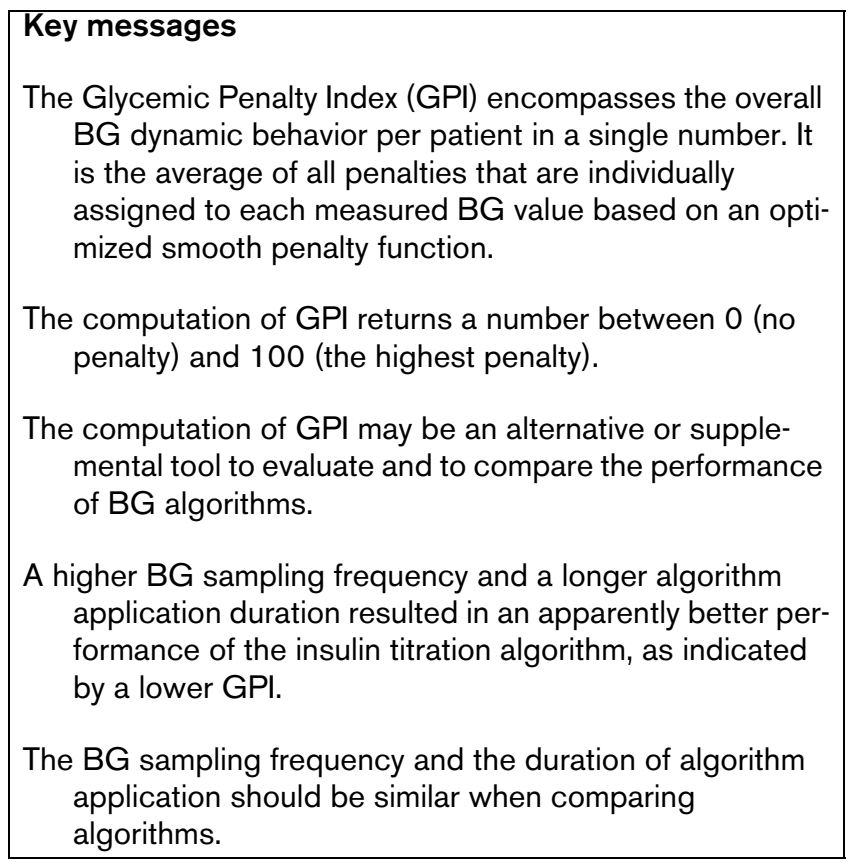

\section{Competing interests}

The authors declare that they have no competing interests.

\section{Authors' contributions}

TVH designed the engineering study, developed the GPI concept, and wrote the manuscript. JDB and MB helped to statistically analyze the data and to draft the manuscript. BDM conceived of the engineering study, and participated in its design and coordination and helped to draft the manuscript. GVdB designed the clinical study, participated in the design of the engineering study and helped to draft the manuscript. All authors read and approved the final manuscript.

\section{Acknowledgements}

The authors thank the nursing staff of the intensive care unit (Katholieke Universiteit Leuven, Belgium) for data sampling, and Pieter Wouters for data acquisition. TVH is a research assistant at the Katholieke Universiteit Leuven. BDM and GVdB are full professors at the Katholieke Universiteit Leuven. The research was supported by: Research Council KUL: GOA/2007/14, GOA AMBioRICS, CoE EF/05/006, IOFSCORES4CHEM, several PhD/postdoc and fellow grants; Flemish
Government: FWO: PhD/postdoc grants, projects G.0452.04, G.0499.04, G.0211.05, G.0226.06, G.0321.06, G.0302.07, G.0320.08, G.0558.08, G.0557.08, research communities (ICCoS, ANMMM, MLDM); IWT: PhD Grants, McKnow-E, Eureka-Flite+; Belgian Federal Science Policy Office: IUAP P6/04; EU: ERNSI; Contract Research: AMINAL.

\section{References}

1. Wolfe R, Allsop J, Burke J: Glucose metabolism in man: responses to intravenous glucose infusion. Metabolism 1979, 28:210-220.

2. Wolfe R, Herndon D, Jahoor F, Miyoshi H, Wolfe M: Effect of severe burn injury on substrate cycling by glucose and fatty acids. N Engl J Med 1987, 317:403-408.

3. Shangraw R, Jahoor F, Miyoshi H, Neff W, Stuart C, Herndon D, Wolfe R: Differentiation between septic and postburn insulin resistance. Metabolism 1989, 38:983-989.

4. Capes S, Hunt D, Malmberg K, Gerstein H: Stress hyperglycaemia and increased risk of death after myocardial infarction in patients with and without diabetes: a systematic overview. Lancet 2000, 355:773-778.

5. Capes S, Hunt D, Malmberg K, Pathak P, Gerstein H: Stress hyperglycemia and prognosis of stroke in nondiabetic and diabetic patients: a systematic overview. Stroke 2001, 32:2426-2432.

6. Umpierrez G, Isaacs S, Bazargan N, You X, Thaler L, Kitabchi A: Hyperglycemia: an independent marker of in-hospital mortality in patients with undiagnosed diabetes. J Clin Endocrinol Metab 2002, 87:978-982.

7. Rovlias A, Kotsou S: The influence of hyperglycemia on neurological outcome in patients with severe head injury. Neurosurgery 2000, 46:335-342.

8. Van den Berghe G, Wouters P, Weekers F, Verwaest C, Bruyninckx F, Schetz M, Vlasselaers D, Ferdinande P, Lauwers P, Bouillon R: Intensive insulin therapy in the critically ill patients. $N$ Engl J Med 2001, 345:1359-1367.

9. Van den Berghe G, Wilmer A, Hermans G, Meersseman W, Wouters P, Milants I, Van Wijngaerden E, Bobbaers $H$, Bouillon R: Intensive insulin therapy in the medical ICU. $N$ Engl $\mathrm{J} \mathrm{Med}$ 2006, 354:449-461.

10. Krinsley J: Effect of an intensive glucose management protocol on the mortality of critically ill adult patients. Mayo Clin Proc 2004, 79:992-1000.

11. Van den Berghe G, Wouters $P$, Bouillon R, Weekers F, Verwaest C, Schetz M, Vlasselaers D, Ferdinande P, Lauwers P: Outcome benefit of intensive insulin therapy in the critically ill: Insulin dose versus glycemic control. Crit Care Med 2003, 31:359-366.

12. Goldberg P, Siegel M, Sherwin R, Halickman J, Lee M, Bailey V Lee S, Dziura J, Inzucchi S: Implementation of a safe and effective insulin infusion protocol in a medical intensive care unit. Diabetes Care 2004, 27:461-467.

13. Taylor B, Schallom M, Sona C, Buchman T, Boyle W, Mazuski J, Schuerer D, Thomas J, Kaiser C, Huey W, Ward MR, Zack JE, Coopersmith CM: Efficacy and safety of an insulin infusion protocol in a surgical ICU. J Am Coll Surg 2006, 202:1-9.

14. Brown $G$, Dodek $P$ : Intravenous insulin nomogram improves blood glucose control in the critically ill. Crit Care Med 2001, 29:1714-1719.

15. Chase J, Shaw G, Wong X, Lotz T, Lin J, Hann C: Model-based glycaemic control in critical care - a review of the state of the possible. Biomed Sig Proc Control 2006, 1:3-21.

16. Chase J, Shaw G, Hann C, LeCompte A, Lonergan T, Willacy M, Wong XW, Lin J, Lotz T: Clinical validation of a model-based glycaemic control design approach and comparison to other clinical protocols. Proceedings of the 28th IEEE EMBS Annual International Conference (EMBC 06) 2006:59-62. 28 ${ }^{\text {th }}$ Annual International Conference of the IEEE Engineering in Medicine and Biology Society. New York City, New York, USA. Publisher: IEEE Engineering in Medicine and Biology (Piscateway, New Jersey, USA)

17. Lonergan T, Compte A, Willacy M, Chase J, Shaw G, Hann C, Lotz T, Lin J, Wong XW: A pilot study of the SPRINT protocol for tight glycemic control in critically III patients. Diabetes Technol Ther 2006, 8:449-462. 
18. Wong XW, Chase JG, Shaw GM, Hann CE, Lotz T, Lin J, SinghLevett I, Hollingsworth LJ, Wong OSW, Andreassen S: Model predictive glycaemic regulation in critical illness using insulin and nutrition input: a pilot study. Med Eng Phys 2006, 28:665-681.

19. Hovorka R: Continuous glucose monitoring and closed-loop systems. Diabet Med 2006, 23:1-12.

20. Hovorka R, Kremen J, Blaha J, Matias M, Anderlova K, Bosanska L, Roubicek T, Wilinska M, Chassin L, Svacina S, Haluzik M: Blood glucose control by a model predictive control algorithm with variable sampling rate versus a routine glucose management protocol in cardiac surgery patients: a randomized controlled trial. J Clin Endocrinol Metab 2007, 92:2960-2964.

21. Plank J, Blaha J, Cordingley J, Wilinska M, Chassin L, Morgan C, Squire S, Haluzik M, Kremen J, Svacina S, Toller W, Plasnik A, Ellmerer M, Hovorka R, Pieber TR: Multicentric, randomized, controlled trial to evaluate blood glucose control by the model predictive control algorithm versus routine glucose management protocols in intensive care unit patients. Diabetes Care 2006, 29:271-276.

22. Vogelzang M, Zijlstra F, Nijsten MW: Design and implementation of GRIP: a computerized glucose control system at a surgical intensive care unit. BMC Med Inform Decis Mak 2005, 5:38.

23. Van Herpe T, Haverbeke N, Pluymers B, Van den Berghe G, De Moor B: The application of model predictive control to normalize glycemia of critically ill patients. Proceedings of the European Control Conference 2007 (ECC 07) 2007:3116-3123. European Union Control Association and Institute of Communication and Computer Systems of National Technical University of Athens (NTUA)

24. Haverbeke N, Van Herpe T, Diehl M, Van den Berghe G, De Moor $\mathrm{B}$ : Nonlinear model predictive control with moving horizon state and disturbance estimation - application to the normalization of blood glucose in the critically ill. Accepted for publication in Proceedings of the 17th IFAC World Congress (IFAC WC 2008) 2008.

25. Chee F, Fernando T, van Heerden P: Closed-loop glucose control in critically ill patients using continuous glucose monitoring system (CGMS) in real time. IEEE Trans Inf Technol Biomed 2003, 7:43-53.

26. Chee F, Fernando T, Savkin A, van Heerden V: Expert PID control system for blood glucose control in critically ill patients. IEEE Trans Inf Technol Biomed 2003, 7:419-425.

27. Meynaar I, Dawson L, Tangkau P, Salm E, Rijks L: Introduction and evaluation of a computerised insulin protocol. Intensive Care Med 2007, 33:591-596.

28. Vogelzang M, van der Horst I, Nijsten M: Hyperglycaemic index as a tool to assess glucose control: a retrospective study. Crit Care 2004, 8:122-127.

29. Van den Berghe G: How to compare adequacy of algorithms to control blood glucose in the intensive care unit? Crit Care 2004, 8:151-152.

30. Hovorka R, Wilinska M, Chassin L, Dunger D: Roadmap to the artificial pancreas. Diabetes Res Clin Pract 2006, 74:S178-S182.

31. International Organisation for Standardization: ISO 15197 IOfS: in vitro diagnostic test systems - requirements for blood-glucose monitoring systems for self-testing in managing diabetes mellitus. Geneva, Switzerland: International Organisation for Standardization; 2003.

32. Cox D, Clarke W, Gonder-Frederick L, Pohl S, Hoover C, Snyder A, Zimbelman L, Carter W, Bobbitt S, Pennebaker J: Accuracy of perceiving blood glucose in IDDM. Diabetes Care 1985, 8:529-536.

33. Clarke W, Cox D, Gonder-Frederick L, Carter W, Pohl S: Evaluating clinical accuracy of systems for self-monitoring of blood glucose. Diabetes Care 1987, 10:622-628.

34. Mackenzie I, Ingle S, Zaidi S, Buczaski S: Hypoglycaemia? So what! Intensive Care Med 2006, 32:620-621.

35. Van den Berghe G: Textbook of Critical Care Medicine 5th edition. Philadelphia, PA: Elsevier-Saunders; 2005:82-86.

36. Maddala G: Introduction to Econometrics 3rd edition. Chichester, UK: Wiley; 2001

37. Krinsley J: Association between hyperglycemia and increased hospital mortality in a heterogeneous population of critically ill patients. Mayo Clin Proc 2003, 78:1471-1478.
38. Grey N, Perdrizet G: Reduction of nosocomial infections in the surgical intensive-care unit by strict glycemic control. Endocr Pract 2004, 10(Suppl 2):46-52.

39. Vanhorebeek I, Van den Berghe G: Diabetes of injury: novel insights. Endocrinol Metab Clin North Am 2006, 35:859-872.

40. Vanhorebeek I, Langouche L, Van den Berghe G: Intensive insulin therapy in the intensive care unit: update on clinical impact and mechanisms of action. Endocr Pract 2006, 12(Suppl 3):14-22.

41. Roth J, Bolin B, Baird R: Evaluation of blood glucose values in critically ill patients before and after implementation of an intensive insulin infusion protocol. Proc Bayl Univ Med Cent 2007, 20:237-239.

42. Knaus W, Draper E, Wagner D, Zimmerman J: APACHE II: a severity of disease classification system. Crit Care Med 1985 13:818-829.

43. Cox D, Gonder-Frederick L, Kovatchev B, Julian D, Clarke W: Understanding error grid analysis. Diabetes Care 1997, 20:911-912.

44. Egi M, Bellomo R, Stachowski E, French C, Hart G, Stow P: Circadian rhythm of blood glucose values in critically ill patients. Crit Care Med 2007, 35:416-421.

45. Van den Berghe G, Wilmer A, Milants I, Wouters P, Bouckaert B, Bruyninckx $F$, Bouillon R, Schetz M: Intensive insulin therapy in mixed medical/surgical intensive care units: benefit versus harm. Diabetes 2006, 55:3151-3159. 